\title{
Postbuckling behavior and imperfection sensitivity of elastic structures by the Lyapunov-Schmidt-Koiter approach
}

\author{
Ralf Peek and Mostafa Kheyrkhahan \\ College of Engineering, The University of Michigan, Ann Arbor, MI 48109-2125, USA
}

Received 27 February 1992

\begin{abstract}
Beginning with the work of Koitcr in 1945, valuable insights into the postbuckling behavior of structures have been gained by Lyapunov-Schmidt decomposition of the displacements followed by an asymptotic expansion about the bifurcation point. Here this methodology is generalized to include nonlinear prebuckling behavior, as well as multiple, not necessarily coincident buckling modes. The expansion of the reduced equilibrium equations is performed about a reference point (which need not coincide with any of the bifurcation points), and applies no matter whether the modes are coincident, closely spaced, or well separated. From a variety of possible decompositions of the admissible space of displacements, two are incorporated into a finite element program. Theoretical considerations, and numerical examples in which asymptotic results are compared to 'exact' results, indicate that one of the decompositions has some important advantages over the other. Examples include a shallow arch, and a beam on elastic foundation problem exhibiting symmetry-breaking modal interaction.
\end{abstract}

\section{Introduction}

Certain structures, and especially those with multiple coincident buckling modes, can be very sensitive to imperfections. For such structures, even a small imperfection can produce a considerable drop in load carrying capacity. More precisely, the load at the first limit point of the imperfect structure is considerably lower than the bifurcation load of the perfect structure. Perhaps the best known example of this is the thin cylinder under axial compression [1-5], but imperfection sensitivity can also occur for other structures, such as domes, thin-walled beams, and ribbed shells and plates where both global and local modes are possible. Optimization of structures also tends to produce coincident buckling modes, with the possibility of symmetry-breaking modal interactions.

Methods to calculate the buckling loads for the perfect structure are well established and available in most general purpose codes. They do not provide any information in regard to postbuckling behavior and imperfection sensitivity, however. A second approach, one that allows the effect of imperfections to be included, is based on numerically tracking the equilibrium path of the imperfect structure beyond the point of maximum load (limit point). This can be achieved by a change in the 'loading' parameter. For example, an approximation to the arclength along the equilibrium branch might be controlled at every loadstep, instead of the magnitude of the applied loads [6-8]. This method is very useful and also available in most general purpose nonlinear codes, but it requires a separate analysis for each magnitude and shape of imperfection to be considered. Also equilibrium paths may have strong curvatures in the vicinity of bifurcation points, rendering them difficult to track numerically.

A third approach, the one further explored here, is based on the Lyapunov-Schmidt decomposition together with an asymptotic expansion about the bifurcation point. This is the approach used by Koiter [1] in his pioneering work. It is applicable to elastic structures with a potential energy function. Its advantage is that it provides more insight into the nature of the bifurcation, and an approximate drop in load-carrying capacity can readily be calculated for a variety of shapes and magnitudes of imperfections

Correspondence to: Dr. Ralf Peek, Department of Civil Engineering, 2340 GG Brown Lab., University of Michigan, Ann Arbor, MI 48109-2125, USA. 
with minimal effort. Thus it is also possible to find the worst shape of imperfection (i.e. the imperfection shape that produces the largest load drop for a given magnitude of imperfection) by finding the bifurcated branch for the perfect structure on which the load drops most rapidly [9-11].

This Lyapunov-Schmidt-Koiter (LSK) approach to the postbuckling behavior and imperfection sensitivity of structures has been extensively used in analytical studies for specific structural geometries [12-18]. However, the implementation of this methodology in finite element programs that could be used for general structural geometries is still in the early stages of its development [19-26].

In this paper, the general theory underlying the LSK method is reviewed, and generalized to obtain an expansion of the reduced equilibrium equations that is valid for nonlinear prebuckling behavior, as well as non-coincident modes. Although problems are addressed in $[1,13]$ with nonlinear prebuckling behavior and coincident modes, and Byskov and Hutchinson [27] address problems with noncoincident modes but linear prebuckling behavior, an asymptotic expansion of the reduced equilibrium equations that is valid for noncoincident modes and nonlinear prebuckling behavior appears not to be available to date. Here the expansion is performed about a reference point which need not coincide with any of the bifurcation points. This gives rise to corrections to the mode shapes which vanish when the modes are fully coincident and the expansion is performed about the multiple bifurcation point. Another feature of this presentation is that the expansions are continued one term beyond the leading order terms for an asymmetric bifurcation. These higher order terms become important in evaluating the merits of different decompositions of the displacements.

From a wide range of possible decompositions of the space of admissible displacements $A$, two are implemented and evaluated. In both cases $A$ is decomposed into a space $A_{0}$ spanned by the buckling modes (eigenvectors of the stability matrix corresponding to the lowest eigenvalues), and a complementary space $\hat{A}$. It is shown both theoretically, and by numerical examples, that choosing $\hat{A}$ to be the set of all displacements $\hat{u}$ that are orthogonal to the buckling modes (with respect to a linear combination of the tangent stiffness matrix and the rate of change of the tangent stiffness matrix along the principal branch) has certain advantages over other choices of the complementary space $\hat{A}$. In the authors' opinion, these advantages outweigh the slight additional costs of this decomposition in computational and programming effort.

Finally a number of illustrative examples involving nonlinear prebuckling behavior as well as symmetry breaking mode interaction are presented, and the results from the asymptotic analysis are compared to 'exact' results obtained by numerically tracking the bifurcated equilibrium branches. The accuracy of the asymptotic results for the drop in load carrying capacity caused by an imperfection is also examined in these examples.

\section{Theory}

Suppose that (i) the structure considered is elastic with a sufficiently smooth potential energy function $\phi=\phi(u, \lambda, \bar{u})$, where $u$ is the displacement field, $\lambda$ is the load parameter, $\bar{u}$ represents an imperfection with $\bar{u}=0$ corresponding to the perfect structure; (ii) the sets of admissible displacement and imperfection fields, $A$ and $\vec{A}$, respectively, are normed vector spaces, and (iii) a smooth principal solution $u(\lambda)$ exists which vanishes at $\lambda=0$ and satisfies the equilibrium condition

$$
\phi{ }_{u}(\stackrel{0}{u}(\lambda), \lambda, 0) \delta u=0 \quad \forall \delta u \in A, \forall \lambda,
$$

where $(\cdot)_{u}$ denotes a Gateaux (or Frechet) derivative.

The analysis proceeds by choosing a reference point on the principal equilibrium branch about which the asymptotic expansion is to be performed. The value of the load parameter $\lambda$ at the reference point is denoted by $\lambda_{c}$. In contrast to the classical analysis [1] in which the expansion is performed about a multiple bifurcation point, here the reference point need not coincide with any of the bifurcation points. Any point on the principal equilibrium branch can be used as the reference point about which the expansion is performed. This expansion is valid in some neighborhood of the reference point no matter whether the modes are fully coincident, closely spaced, or well separated. 
Following the spirit of the Lyapunov-Schmidt approach, the space of admissible displacements $A$ is decomposed into a subspace $A_{0}$, which is spanned by a finite number of modes $\vec{i}$, and a complementary space $\hat{A}$ such that

$$
A=A_{0} \oplus \hat{A}, \quad A_{0} \cap \hat{A}=\{0\},
$$

and there exists an $\alpha>0$ such that

$$
\phi^{c}{ }^{c} \delta u \hat{u} \delta \hat{u} \geqslant \alpha \quad \forall \delta \hat{u} \in \hat{A} \quad \text { with }\|\delta \hat{u}\|=1 .
$$

Equations (2) ensure that any displacement field $u \in A$ can be decomposed uniquely as

$$
u=u_{0}+\hat{u}, \quad u_{0} \in A_{0}, \quad \hat{u} \in \hat{A},
$$

whereas (3) ensures positive definiteness of the stability operator $\phi_{\text {.uu }}$ restricted to $\hat{A}$ in a nonvanishing neighborhood of the reference point. For a finite-dimensional space of displacements $A$, condition (3) follows from (2), if the stability operator $\phi_{{ }^{\prime}}^{c}$ is positive semidefinite and $A_{0}$ contains its nullspace.

The space $\hat{A}$ is often taken to be the orhogonal complement of $A_{0}$, but, as was observed by Thompson and Hunt [28], it is not necessary to introduce this restriction. Other possibilities can lead to simpler implementations of the method.

Considerable flexibility also exists in the choice of the space $A_{0}$. For the case of fully coincident modes at $\lambda=\lambda_{c}$, the natural choice for $A_{0}$ is the nullspace of the stability operator evaluated at the reference point. In this case, the basis vectors $\tilde{u}$ satisfy

$$
\phi{ }^{c}{ }_{u u}^{i} \tilde{u} \delta u=0 \quad \forall \delta u \in A
$$

where a superscript $c$ applied to the potential energy or any of its derivatives denotes evaluation at the reference point, $(u, \lambda, \bar{u})=\left(\stackrel{0}{u}\left(\lambda_{c}\right), \lambda_{c}, 0\right)$. If the buckling modes are not fully coincident $\tilde{\tilde{u}}$ can be chosen as the eigenvectors corresponding to the smallest eigenvalues $\mu_{i}$ in

$$
\left(\phi^{c}{ }_{u u}+\mu_{i} \dot{\phi}_{{ }_{u u}}\right) \stackrel{i}{\tilde{u}} \delta u=0 \quad \forall \delta u \in A,
$$

where a dot placed above any entity denotes evaluation of the entity on the principal branch for the perfect structure followed by differentiation with respect to $\lambda$ and evaluation at $\lambda=\lambda_{c}$. Thus,

$$
\dot{\phi}_{{ }_{u u}} \equiv\left[\frac{\mathrm{d}}{\mathrm{d} \lambda} \phi_{\cdot_{u u}}(\stackrel{0}{u}(\lambda), \lambda, 0)\right]_{\lambda=\lambda_{c}}=\phi_{{ }_{u u u}^{c}}^{\mathrm{u}} \dot{u}+\phi_{{ }_{u u \lambda}^{c}}^{c}, \quad \dot{u}=\left[\frac{\mathrm{d} u}{\mathrm{~d} \lambda}\right]_{\lambda=\lambda_{c}} .
$$

More generally the modes $\stackrel{i}{\tilde{u}}$ need neither be chosen from (5) nor from (6). Any set of linearly independent vectors can be chosen, as long as conditions (2) and (3) are satisfied.

Applying the decomposition of (4), the displacements can be written as

$$
u=\stackrel{0}{u}(\lambda)+\sum_{i} \xi_{i} \stackrel{i}{u}+\hat{u}, \quad \hat{u} \in \hat{A},
$$

where $\xi_{i}$ are scalar buckling deflections. Correspondingly, the equilibrium conditions can be decomposed into the following two conditions:

$$
\begin{aligned}
& \phi \cdot_{u}\left(\stackrel{0}{u}(\lambda)+\sum_{j} \xi_{j} \tilde{j} \tilde{u}+\hat{u}, \lambda, \varepsilon \overline{\bar{u}}\right) \delta \hat{u}=0 \quad \forall \delta \hat{u} \in \hat{A}, \\
& \phi \cdot_{u}\left(\stackrel{0}{u}(\lambda)+\sum_{j} \xi_{j} \tilde{u}+\hat{u}+\lambda, \varepsilon \overline{\bar{u}}\right) \tilde{u}=0 \quad \forall i .
\end{aligned}
$$


In (9) and (10), the imperfection has been written as $\bar{u}=\varepsilon \overline{\bar{u}}$, where $\overline{\bar{u}} \in \bar{A}$ is a normalized imperfection shape, $\varepsilon$ is a scalar magnitude of the imperfection.

In view of (3) and the implicit function theorem, (9) admits a unique solution $\hat{u}=\hat{u}\left(\xi_{i}, \lambda, \varepsilon\right) \in \hat{A}$ for any given $\left(\xi_{i}, \lambda, \varepsilon\right)$ in the vicinity of the reference point. The corresponding total displacement can be written as

$$
u=u\left(\xi_{i}, \lambda, \varepsilon\right) \equiv \stackrel{0}{u}(\lambda)+\sum_{j} \xi_{j} \tilde{u}+\hat{u}\left(\xi_{i}, \lambda, \varepsilon\right),
$$

and the set of all such solutions is referred to as the partial equilibrium surface ${ }^{1}$ (since equilibrium conditions are partially satisfied on this surface), whereas $\left(\xi_{i}, \lambda, \varepsilon\right)$ are referred to as the parameters of the partial equilibrium surface. This partial equilibrium surface is not a property of the structure; it depends on the choice of the space $\hat{A}$.

It follows by differentiation of (11) that

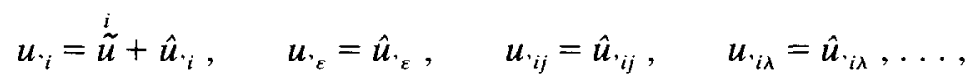

where $(\cdot)_{i} \equiv \partial(\cdot) / \partial \xi_{i}$. Also, since $\hat{u} \in \hat{A}$, the derivatives of $\hat{u}$ must also lie in the complementary space $\hat{A}$. Thus $u_{{ }_{\varepsilon}}, u_{r_{i j}}, u_{{ }_{i \lambda}}$ and their derivatives are in $\hat{A}$. For the subsequent analysis, it is convenient to introduce the following notation for the derivatives of the displacements with respect to parameters of the partial equilibrium surface evaluated at the reference point:

$$
\begin{aligned}
& \stackrel{(i)}{u} \equiv\left[u_{i}\right]_{\left(\xi_{1}, \lambda, \varepsilon\right)=\left(0, \lambda_{c}, 0\right)}, \quad \stackrel{(\varepsilon)}{u} \equiv\left[u_{\cdot_{\varepsilon}}\right]_{\left(\xi_{i}, \lambda, \varepsilon\right)=\left(0, \lambda_{c}, 0\right)}, \quad \stackrel{(i)}{u} \equiv\left[u_{\cdot_{i j}}\right]_{\left(\xi_{i}, \lambda, \varepsilon\right)=\left(0, \lambda_{c}, 0\right)}, \ldots \\
& \stackrel{(i)}{\hat{u}} \equiv\left[\hat{u}_{i}\right]_{\left(\xi_{i}, \lambda, \varepsilon\right)=\left(0, \lambda_{c}, 0\right)}, \quad \stackrel{(\varepsilon)}{\hat{u}} \equiv\left[\hat{u}_{\varepsilon}\right]_{\left(\xi_{i}, \lambda, \varepsilon\right)=\left(0, \lambda_{c}, 0\right)}, \quad \stackrel{(i j)}{\hat{u}} \equiv\left[\hat{u}_{i j}\right]_{\left(\xi_{i}, \lambda, \varepsilon\right)=\left(0, \lambda_{c}, 0\right)}, \ldots
\end{aligned}
$$

With the aid of (11), (9) can be rewritten as

$$
\phi_{u}\left(u\left(\xi_{i}, \lambda, \varepsilon\right), \lambda, \varepsilon \overline{\bar{u}}\right) \delta \hat{u}=0 \quad \forall \delta \hat{u} \in \hat{A}, \forall\left(\xi_{i}, \lambda, \varepsilon\right) .
$$

Taking derivatives of this equation with respect to $\xi_{\imath}, \lambda$ and $\varepsilon$ and then evaluating the results at the reference point, produces

$$
\begin{aligned}
& (\ldots)_{i} \Rightarrow \phi_{i_{u u}^{c}}^{c} \stackrel{(i)}{u} \delta \hat{u}=0 \quad \forall \delta \hat{u} \in \hat{A}, \\
& (\ldots)_{\lambda} \Rightarrow\left(\phi^{c}{ }_{u u}^{(\lambda)} \stackrel{(\lambda)}{u}+\phi_{{ }_{u \lambda}}^{c}\right) \delta \hat{u}=0 \quad \forall \delta \hat{u} \in \hat{A}, \\
& (\ldots)_{\varepsilon} \Rightarrow\left(\phi^{c}{ }_{u u}{ }^{(\varepsilon)} u+\phi^{c}{ }_{u \bar{u}}^{c} \overline{\bar{u}}\right) \delta \hat{u}=0 \quad \forall \delta \hat{u} \in \hat{A}, \\
& (\ldots)_{\imath j} \Rightarrow\left(\phi_{u u}^{c} \stackrel{(i j)}{u}+\phi_{\cdot u u u}^{c} \stackrel{(i)}{u} \stackrel{(j)}{u}\right) \delta \hat{u}=0 \quad \forall \delta \hat{u} \in \hat{A} \\
& (\ldots)_{i \lambda} \Rightarrow\left(\phi_{{ }^{c}}^{c}{ }_{u u} \stackrel{(i \lambda)}{u}+\dot{\phi}_{u u} \stackrel{(i)}{u}\right) \delta \hat{u}=0 \quad \forall \delta \hat{u} \in \hat{A} \text {, } \\
& (\ldots)_{i \varepsilon} \Rightarrow\left\{\phi_{\urcorner_{u u}^{c}}^{c} \stackrel{(i \varepsilon)}{u}^{(i)}+\left(\phi_{{ }^{u u u}} \stackrel{(\varepsilon)}{u}+\phi_{{ }^{u} u \bar{u}} \overline{\bar{u}}\right) \stackrel{(i)}{u}\right\} \delta \hat{u}=0 \quad \forall \delta \hat{u} \in \hat{A}, \\
& (\ldots)_{\lambda \varepsilon} \Rightarrow\left(\phi^{c}{ }_{u u} \stackrel{(\lambda \varepsilon)}{u}+\dot{\phi}_{{ }_{u u}} \stackrel{(\varepsilon)}{u}+\dot{\phi}_{{ }_{u \bar{u}}} \overline{\bar{u}}\right) \delta \hat{u}=0 \quad \forall \delta \hat{u} \in \hat{A}, \\
& (\ldots)_{i j k} \Rightarrow\left\{\phi_{u u}^{c} \stackrel{(i j k)}{u}^{u}+\phi_{\cdot u u u}^{c}(\stackrel{(i)}{u} \stackrel{(j k)}{u}+\stackrel{(j)}{u} \stackrel{(i k)}{u}+\stackrel{(k)}{u} \stackrel{(i j)}{u})\right. \\
& \left.+\phi_{\text {:uuuu }}^{c} \stackrel{(i)}{u} \stackrel{(1)}{u} \stackrel{(k)}{u}\right\} \delta \hat{u}=0 \quad \forall \delta \hat{u} \in \hat{A},
\end{aligned}
$$

\footnotetext{
'Thompson and Hunt [28] call this the 'surface of activity'.
} 


$$
\begin{aligned}
& (\ldots)_{i j \lambda} \Rightarrow\left\{\phi_{v_{u u}^{c}} \stackrel{(i j \lambda)}{u}+\dot{\phi}_{\cdot u u} \stackrel{(i j)}{u}+\dot{\phi}_{\cdot u u u} \stackrel{(i)}{u} \stackrel{(j)}{u}\right. \\
& +\phi^{c}{ }^{c}(\stackrel{(i \lambda)}{u}(\stackrel{(j)}{u}+\stackrel{(j \lambda)(i)}{u} \stackrel{(i)}{u})\} \delta \hat{u}=0 \quad \forall \delta \hat{u} \in \hat{A}, \\
& (\ldots)_{i \lambda \lambda} \Rightarrow\left\{\phi_{{ }_{u u}^{c}} \stackrel{(i \lambda \lambda)}{u}+2 \dot{\phi}_{u u} \stackrel{(i \lambda)}{u}+\ddot{\phi}_{u u} \stackrel{(i)}{u}\right\} \delta \hat{u}=0 \quad \forall \delta \hat{u} \in \hat{A}
\end{aligned}
$$

It follows from (16), (12) and (13) that

$$
\stackrel{(i)}{u}=\stackrel{i}{\tilde{u}}+\stackrel{(i)}{\hat{u}}, \quad \phi^{c}{ }_{u u}(\stackrel{i}{\tilde{u}}+\stackrel{(i)}{\hat{u}}) \delta \hat{u}=0 \quad \forall \delta \hat{u} \in \hat{A} .
$$

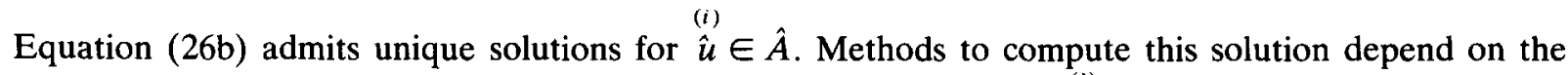
choice of $\hat{A}$ and are discusscd later. Once this solution is available, $\stackrel{(i)}{u}$ can be calculated from (26a).

To obtain $\stackrel{(\lambda)}{u}$ from (17), differentiate (1) with respect to lambda and evaluate at $\lambda=\lambda_{c}$ to obtain

$$
\left(\phi^{c}{ }_{u u} \dot{u}+\phi_{{ }_{u \lambda}}^{c}\right) \delta u=0 \quad \forall \delta u \in A .
$$

Comparing this result with (17), and invoking uniqueness of the solution to (17), and also noting from differentiation of (11) with respect to $\lambda$ that $\stackrel{(\lambda)}{u}=\dot{u}+\stackrel{(\lambda)}{\hat{u}}$, it is seen that

$$
\stackrel{(\lambda)}{u}=\dot{u}, \quad \stackrel{(\lambda)}{\hat{u}}=0 .
$$

Indeed, by substituting $\xi_{i}=\varepsilon=0$ into (9), comparing the result with (1), and invoking the uniqueness of the solutions for $\hat{u}$, it is seen that

$$
\hat{u}(0, \lambda, 0)=0 \quad \forall \lambda \Rightarrow \stackrel{(\lambda)}{\hat{u}}=\stackrel{(\lambda \lambda)}{\hat{u}}=\cdots=0 .
$$

Since $\stackrel{(\varepsilon)}{u}, \stackrel{(i j)}{u}, \stackrel{(i \lambda)}{u}, \stackrel{(i \varepsilon)}{u}, \stackrel{(i j k)}{u}, \stackrel{(i j \lambda)}{u}$ and $\stackrel{(i \lambda \lambda)}{u}$ are in the space $\hat{A}$, these quantities can also be determined uniquely from (18)-(25). Higher derivatives of $u\left(\xi_{i}, \lambda, \varepsilon\right)$ can also be evaluated in a similar fashion to obtain the following expansion for the displacements on the partial equilibrium surface:

$$
u\left(\xi_{i}, \lambda, \varepsilon\right)=\stackrel{0}{u}(\lambda)+\sum_{i} \xi_{i} \stackrel{(i)}{u}+\Delta \lambda \dot{u}+\varepsilon \stackrel{(\varepsilon)}{u}+\frac{1}{2} \sum_{i, j} \xi_{i} \xi_{j} \stackrel{(i j)}{u}+\Delta \lambda \sum_{i} \xi_{i} \stackrel{(i \lambda)}{u}+\cdots
$$

Consider now the second equilibrium condition, (10). If a reduced potential energy function is defined as

$$
\psi\left(\xi_{i}, \lambda, \varepsilon\right) \equiv \phi\left(u\left(\xi_{i}, \lambda, \varepsilon\right), \lambda, \varepsilon \overline{\bar{u}}\right),
$$

and this is differentiated with respect to $\xi_{i}$, one obtains

$$
\psi_{{ }_{i}}=\phi_{\cdot_{u}}\left(\stackrel{i}{\tilde{u}}+\hat{u}_{{ }_{i}}\right)=\phi_{{ }_{u}} \stackrel{i}{\tilde{u}} .
$$

The second equality in (32) follows from (9), since $\phi_{{ }_{u}}$ is evaluated on the partial equilibrium surface, and $\hat{u}$ as well as its derivatives lie in the complementary space $\hat{A}$. Thus (10) is seen to be equivalent to stationarity of the reduced potential energy $\left(\psi_{i}=0\right)$. In other words, stationarity of the reduced potential energy is a necessary and sufficient condition for equilibrium. It can also be shown [11] that equilibrium states are stable if and only if the matrix $\psi_{i j}$ is positive definite.

Expanding (32) about the reference point produces

$$
\begin{aligned}
\psi_{i}= & \phi_{i \varepsilon} \varepsilon+\sum_{j} \phi_{i j} \xi_{j}+\sum_{j} \phi_{i j \lambda} \xi_{j} \Delta \lambda+\frac{1}{2} \sum_{j, k} \phi_{i j k} \xi_{j} \xi_{k}+\sum_{j} \phi_{i j \varepsilon} \xi_{j} \varepsilon+\phi_{i \varepsilon \lambda} \varepsilon \Delta \lambda+\frac{1}{6} \sum_{j, k, l} \phi_{i j k l} \xi_{j} \xi_{k} \xi_{l} \\
& +\frac{1}{2} \sum_{j, k} \phi_{i j k \lambda} \xi_{j} \xi_{k} \Delta \lambda+\frac{1}{2} \sum_{j} \phi_{i j \lambda \lambda} \xi_{j} \Delta \lambda^{2}+\cdots=0
\end{aligned}
$$


where $\Delta \lambda \equiv \lambda-\lambda_{c}$, and the postbuckling coefficients $\phi_{i \varepsilon}, \phi_{i j}, \phi_{i j \lambda}, \phi_{i j k}$, etc. are derivatives of the reduced potential energy evaluated at the reference point. Note that $\phi_{i \lambda}=\phi_{i \lambda \lambda}=\phi_{i \lambda \lambda \lambda}=\cdots=0$, since it follows from (31) and (32) that $\psi_{i_{i}}(0, \lambda, 0)=\phi_{{ }_{u}}(\stackrel{0}{u}(\lambda), \lambda, 0) \tilde{u}=0$ for all $\lambda$. The expressions for the other derivatives of the reduced potential energy can be simplified with the aid of (16), to obtain

$$
\begin{aligned}
& \phi_{i j}=\phi_{r_{u u}^{c}}^{c} \stackrel{(i)}{u} \stackrel{(j)}{u} \\
& \phi_{i \varepsilon}=\phi_{\cdot u \bar{u}}^{c} \overline{\bar{u}} \stackrel{(i)}{u} \text {, } \\
& \phi_{i j k}=\phi_{{ }_{u u u}^{c}}^{c} \stackrel{(i)}{u} \stackrel{(j)}{u} \stackrel{(k)}{u}, \\
& \phi_{i j \lambda}=\dot{\phi}_{u u} \stackrel{(i)}{u} \stackrel{(j)}{u}, \\
& \phi_{i j \varepsilon}=\left(\phi_{\urcorner_{u u u}^{c}}^{c} \stackrel{(\varepsilon)}{u}+\phi_{\cdot u u \bar{u}}^{c} \overline{\bar{u}}\right) \stackrel{(i)}{u} \stackrel{(j)}{u}, \\
& \phi_{i \varepsilon \lambda}=\left(\dot{\phi}_{\cdot u u} \stackrel{(\varepsilon)}{u}+\dot{\phi}_{u \bar{u}} \overline{\bar{u}}\right) \stackrel{(i)}{u},
\end{aligned}
$$

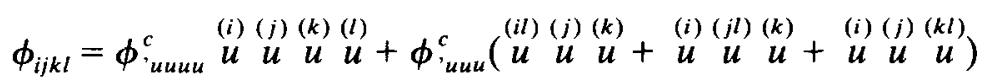

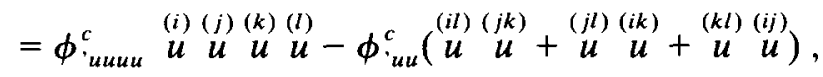

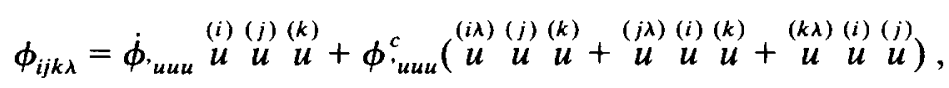

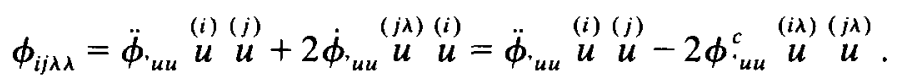

The expansion in (33) is valid no matter whether the modes are coincident, closely spaced, or well separated. However, except for the case of fully coincident modes, the solutions of interest are in general a finite distance away from the reference point. Therefore (33) does not lend itself directly to asymptotic methods of solution.

To overcome this difficulty, the structure must be modified by introducing a mode splitting parameter, $\zeta$, say, such that the modes become fully coincident in the limit as $\zeta \rightarrow 0$. This can be achieved by replacing the potential energy $\phi(u, \lambda, \bar{u})$ by a modified potential energy given by

$$
\hat{\phi}(u, \lambda, \bar{u}, \zeta)=\phi(u, \lambda, \bar{u})+\frac{1}{2}(\zeta-1) \sum_{k, l} c_{k l}(u-\stackrel{0}{u}(\lambda), \stackrel{(k)}{u}) g(u-\stackrel{0}{u}(\lambda), \stackrel{(l)}{u}),
$$

where $g(\cdot, \cdot)$ is a bilinear, symmetric form that satisfies the condition

$$
g(\stackrel{(i)}{u}, \hat{u})=0 \quad \forall i, \forall \hat{u} \in \hat{A},
$$

and for which the matrix of coefficients $g^{i j} \equiv g(\stackrel{(i)}{u}, \stackrel{(j)}{u})$ has an inverse, whose elements are denoted by $g_{i j}$, and $c_{k l}$ are constant coefficients given by

$$
c_{k l}=\sum_{i, j} \phi_{i j} g_{i k} g_{j l}
$$

The following observations can be made when comparing the modified structure with the original one:

(1) For $\zeta=1$, they are identical [i.e. $\hat{\phi}(u, \lambda, \bar{u}, 1)=\phi(u, \lambda, \bar{u})]$.

(2) The fundamental solutions $u(\lambda)$ are identical.

(3) If the eigenvalue problem (6) is written for the modified structure, the modes $\tilde{u}$ for the modified structure would not necessarily coincide with those for the original structure.

(4) All postbuckling coefficients are identical [i.e. $\left.\hat{\phi}_{(\ldots)}=\phi_{(\ldots)}\right]$, except that $\hat{\phi}_{i j}=\zeta \phi_{i j}$. 
In view of the last observation, the reduced equilibrium equations for the modified structure become

$$
\begin{aligned}
& \phi_{i \varepsilon} \varepsilon+\zeta \sum_{j} \phi_{i j} \xi_{j}+\sum_{j} \phi_{i j \lambda} \xi_{j} \Delta \lambda+\frac{1}{2} \sum_{j, k} \phi_{i j k} \xi_{j} \xi_{k}+\sum_{j} \phi_{i j \varepsilon} \xi_{j} \varepsilon+\phi_{i \varepsilon \lambda} \varepsilon \Delta \lambda+\frac{1}{6} \sum_{j, k, l} \phi_{i j k l} \xi_{j} \xi_{k} \xi_{i} \\
& \quad+\frac{1}{2} \sum_{j, k} \phi_{i j k \lambda} \xi_{j} \xi_{k} \Delta \lambda+\frac{1}{2} \sum_{j} \phi_{i j \lambda \lambda} \xi_{j} \Delta \lambda^{2}+\cdots=0
\end{aligned}
$$

which does lend itself to asymptotic methods of solution that are valid for small $\zeta$ (see for example [11]. This leads to series solutions for $\varepsilon, \zeta, \Delta \lambda$ and $\xi_{i}$ as functions of a solution parameter denoted by $\xi$. Whereas a variety of parametrizations are possible, it will be assumed here that $\xi$ is defined such that $\Sigma \xi_{i}^{2}=\mathrm{O}\left(\xi^{2}\right)$.

For an asymmetric bifurcation (i.e. when at least one of the coefficients $\phi_{i j k}$ is nonzero), the solutions are of the form $\varepsilon=\mathrm{O}\left(\xi^{2}\right), \zeta=\mathrm{O}(\xi), \Delta \lambda=\mathrm{O}(\xi)$ and $\xi_{i}=\mathrm{O}(\xi)$. Terms in the series solution of the order indicated above will be referred to as the lowest possible order terms. To calculate these lowest possible order terms, it is sufficient to solve a truncated version of (46), in which only the first four terms of (46) are included. (It is readily verified that other terms in (46) are of higher order.) For the perfect structure with coincident modes, these lowest possible order solutions describe the number of equilibrium branches that emanate from the bifurcation as well as their directions (provided that all lowest possible order solutions are isolated). In order to obtain the next higher order term in the series solutions (e.g. curvatures of the solution paths emanating from the bifurcation), the other terms shown in (46) also need to be considered.

For a symmetric bifurcation (i.e. when all $\phi_{i j k}$ are zero), the solutions are of the form $\xi_{i}=\mathrm{O}(\xi)$, $\Delta \lambda=\mathrm{O}\left(\xi^{2}\right), \varepsilon=\mathrm{O}\left(\xi^{3}\right)$ and $\zeta=\mathrm{O}\left(\xi^{2}\right)$. In this case, calculating the lowest possible order solution requires the $\phi_{i j k l} \xi_{j} \xi_{k} \xi_{l}$ term in (46) to be included in addition to the first four terms in (46). Higher order accuracy in the solution for a symmetric bifurcation would require additional terms that are not given in (46).

The truncated version of (46) that provides the lowest possible order solutions are referred to as the leading order reduced equilibrium equation. The terms and the postbuckling coefficients involved in this equation are referred to as 'leading order terms' and 'leading order postbuckling coefficients', respectively. Thus, for an asymmetric bifurcation, the leading order postbuckling coefficients are $\phi_{i j}$, $\phi_{i \varepsilon}, \phi_{i j \lambda}$ and $\phi_{i j k}$. For a symmetric bifurcation, the leading order coefficients are the same, except that the $\phi_{i j k}$ (which are all zero) are replaced by the $\phi_{i j k l}$.

\section{Implementation}

A summary of the procedure for calculation of the postbuckling coefficients $\phi_{i j}, \phi_{i \varepsilon}, \phi_{i j \lambda}, \phi_{i j k}$ and $\phi_{i j k l}$ is as follows:

(1) Obtain a converged solution on the fundamental branch to be used as a reference point. (This point should be close to any bifurcation points of interest.)

(2) Obtain the displacement rate $\dot{u}$ at this point.

(3) Obtain the corrected modes $\stackrel{u}{u}$ from (26a), after calculating $\hat{u}$ from (26b).

(4) If desired, orthonormalize the modes so that

$$
\phi_{i j \lambda}=-\phi_{0} \delta_{i j}
$$

where $\phi_{0}$ is a positive constant and $\delta_{i j}$ is the Kronecker delta. This operation is performed using the modified Gram-Schmidt orthogonalization procedure described in [29].

(5) Obtain the second order displacements $\stackrel{(i j)}{u}$ from (19).

(6) Calculate the postbuckling coefficients of interest using (34)-(42).

The calculation of the displacement rate $\dot{u}$ (step 4) deserves some further comment: usually this displacement rate can be calculated from

$$
\left(\phi^{c}{ }_{u u} \dot{u}+\phi_{{ }_{u \lambda}}^{c}\right) \delta u=0 \quad \forall \delta u \in A .
$$


However, if the reference point is very close to a bifurcation point, the tangent stiffness matrix $\phi^{c}{ }_{u u}$ is close to singular, and (48) does not produce an accurate solution for $\dot{u}$. Under such circumstances, it is preferable to estimate $\dot{u}$ using the results from previous loadsteps. For this purpose, suppose that the reference point is to be the solution at loadstep $i$. Then the values of $u$ and $\mathrm{d} u / \mathrm{d} \lambda$ at loadstep $i-1$ together with the value of $\stackrel{0}{u}$ at loadstep $i-2$ can be used to obtain an approximation for $\stackrel{0}{u}(\lambda)$ that is quadratic in $\lambda$. This expression can be differentiated with respect to $\lambda$ to obtain the desired approximation for $\mathrm{d} u / \mathrm{d} \lambda$ at loadstep $i$, the reference point. The same approach is also useful when tracking an equilibrium branch, to obtain improved predictor values of the displacements at each loadstep.

Within this general framework, a variety of different decompositions of the space of admissible displaccments $A$ are possible, depending on the choice of the complementary space $\hat{A}$. Two different choices for $\hat{A}$ are evaluated here: the first (decomposition $\mathrm{A}$ ) is simpler, and bears some similarity to the 'equivalence transformation' approach of [26]; the second (decomposition B) employs the orthogonal complement of the space spanned by the buckling modes for $\hat{A}$, and is more akin to the classical approach as presented in [1]. Both methods have been implemented in a finite element program named lskfe.

\subsection{Decomposition $A$}

This method relies on the definition of a number of control degrees of freedom. The other degrees of freedom will be referred to as the passive degrees of freedom. If the total number of degrees of freedom is $N$, and the number of modes to be considered in the analysis is $N_{0}$, then $N_{0}$ control degrees of freedom should be chosen, leaving $N_{1} \equiv N-N_{0}$ passive degrees of freedom. Thus any displacement vector $u$ can be written as $u=(\nu, w)$, where $\nu$ contains the passive degrees of freedom, and $w$ contains the control degrees of freedom. The space $\hat{A}$ is taken to be the set of all displacements $u$ which involve zero displacements for all control degrees of freedom. Thus $u=(\nu, w) \in \hat{A}$ implies that $w=0$.

Recall that the choice of $\hat{A}$ must be such that (2) and (3) are satisfied. Clearly (2a) is satisfied. Equation (2b) requires that the $N_{0} \times N_{0}$ matrix $\tilde{W}$ given below be nonsingular.

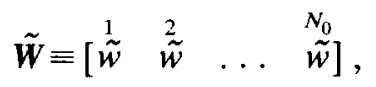

where $\tilde{w}$ denote the controlled components of the modes $\stackrel{i}{\tilde{u}}=(\tilde{i}, \stackrel{i}{\tilde{w}})$. This provides the criterion for an appropriate choice of the control degrees of freedom. It can also be shown (for the finite dimensional case) that the positive definiteness condition (3) is satisfied, provided that the tangent stiffness matrix $\phi_{{ }^{\prime} u u}^{c}$ is positive semidefinite, and the modes ${ }^{i} \tilde{u}$ span the nullspace of $\phi^{c}{ }_{u u}^{c}$.

The main advantage of this choice of the space $\hat{A}$ is that it makes the calculation of the correction to the modes $\stackrel{(i)}{\hat{u}} \in \hat{A}$ from (26b), and the second order displacements $\stackrel{(i j)}{u} \in \hat{A}$ from (19) particularly simple. Only the equations corresponding to the passive degrees of freedom need to be considered in order to calculate the passive components of $\hat{u}$ or $\stackrel{(i j)}{u}$.

More specifically, for the calculation of the corrected buckling modes $\stackrel{(i)}{u}=(\stackrel{(i)}{\nu}, \stackrel{(i)}{w})$ from $(26)$, the passive components of the buckling modes $\tilde{\nu}$ can be taken as zero for simplicity. (They do not affect any of the subsequent results.) Thus $\underset{\tilde{u}}{i}=(0, \tilde{w})=(0, \stackrel{i}{w})$, and $\stackrel{(i)}{\hat{u}}=(\stackrel{(i)}{\hat{\nu}}, 0)=(\stackrel{(i)}{\nu}, 0)$, where $\stackrel{(i)}{\nu}$ is determined from

$$
\left(\phi_{\nu \nu}^{c} \stackrel{(i)}{\nu}+\phi{ }_{\nu}^{c} \underset{\nu w}{\tilde{w}}\right) \delta \nu=0 \quad \forall \delta \nu \in R^{N_{1}}
$$

Here $\phi_{\nu_{\nu}}^{c}$ is an $N_{1} \times N_{1}$ positive definite submatrix of the tangent stiffness matrix $\phi_{{ }_{u u}}^{c}$.

The equation for the second order displacement (19) can be reduced in a similar way to obtain

$$
\left(\phi_{\nu \nu}^{c} \stackrel{(i j)}{\nu}+\phi_{\nu u u}^{c} \stackrel{(i)}{u} \stackrel{(j)}{u}\right) \delta \nu=0 \quad \forall \delta \nu \in R^{N_{1}}
$$


Thus the corrected modes and all second order displacements can be calculated with a single factorization of the reduced tangent stiffness matrix.

Another advantage of this approach is that it is not strictly necessary to solve the eigenvalue problem

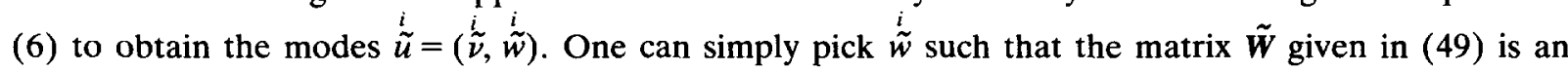
identity matrix. In this case, $\xi_{i}$ represents the displacement for the $i$ th control degree of freedom.

\subsection{Decomposition $B$}

Here the complementary space $\hat{A}$ is taken to be

$$
\hat{A}=\left\{\hat{u} \in \hat{A}:\left(\alpha \phi_{{ }_{u u}}^{c}-\beta \dot{\phi}_{{ }_{u u}}\right) \stackrel{i}{\tilde{u}} \hat{u}=0 \forall i\right\}
$$

where $\alpha$ and $\beta$ are any positive constants (e.g. $\alpha=\beta=1$ ). If in addition, the modes are obtained by solving the eigenvalue problem, (6), then it follows from (6) and (52) that

$$
\left[\begin{array}{cc}
\alpha & -\beta \\
1 & \mu_{i}
\end{array}\right]\left[\begin{array}{cc}
\phi_{{ }_{u u}}^{c} & i \tilde{u} \delta \hat{u} \\
\dot{\phi}_{u u} \tilde{\tilde{u}} \delta \hat{u}
\end{array}\right]=\left[\begin{array}{l}
0 \\
0
\end{array}\right] \forall i, \forall \delta \hat{u} \in \hat{A} .
$$

Thus, as long as $\alpha \mu_{t}+\beta \neq 0$, it follows from (53) that

$$
\phi_{{ }^{c} u u}^{c} \stackrel{i}{\tilde{u}} \delta \hat{u}=\dot{\phi}_{\cdot u u} \tilde{u} \delta \hat{u}=0 \quad \forall \delta \hat{u} \in \hat{A}
$$

Comparing (54) with (16), it is seen that $\stackrel{i}{\tilde{u}}=\stackrel{(i)}{u}$. Thus $\stackrel{(i)}{\hat{u}}=0$, and no correction to the modes is necessary for this case.

Equation (54) leads to a number of simplifications in the evaluation of certain higher order postbuckling coefficients: clearly from (20) and (54), $\stackrel{(i \lambda)}{u}=0$. Thus (39), (41) and (42) reduce to

$$
\phi_{i \varepsilon \lambda}=\dot{\phi} \cdot u \overline{\bar{u}} \stackrel{(i)}{u}, \quad \phi_{i j k \lambda}=\dot{\phi}_{\cdot u u u} \stackrel{(i)}{u} \stackrel{(j)}{u} \stackrel{(k)}{u}, \quad \phi_{i j \lambda \lambda}=\ddot{\phi}_{u u} \stackrel{(i)}{u} \stackrel{(j)}{u}
$$

Further simplifications are possible for a wide class of problems involving linear prebuckling behavior described in Appendix A. In this case, $(55 a-c)$ reduce to

$$
\phi_{i \varepsilon \lambda}=\phi_{i \varepsilon} / \lambda_{c}, \quad \phi_{i j k \lambda}=0, \quad \phi_{i j \lambda \lambda}=0 .
$$

Thus for the perfect structure with an asymmetric bifurcation including only the $\phi_{i j k l} \xi_{i} \xi_{j} \xi_{k}$ terms in addition to the leading order terms does produce a solution of higher order accuracy.

To obtain the solution for the second order displacements, (19) and the condition $\stackrel{(i j)}{u} \in \hat{A}$ can be written as

$$
\left[\begin{array}{cc}
\phi^{c} & b \\
b^{\mathrm{t}} & 0
\end{array}\right]\left[\begin{array}{c}
(i j) \\
u \\
\boldsymbol{a}
\end{array}\right]=\left[\begin{array}{c}
-\phi_{\cdot_{u u u}} \stackrel{(i)}{u} \stackrel{(j)}{u} \\
0
\end{array}\right],
$$

where $\phi^{c}{ }_{u u}$ and $\dot{\phi}_{u u}$ denote $N \times N$ matrices, $\phi_{{ }^{\prime}}^{c} \stackrel{(i) u}{u} \stackrel{(j)}{u}$ denotes an $N \times 1$ column vector, $\boldsymbol{a}$ is an $N_{0} \times 1$ column vector, and $\boldsymbol{b}$ is an $N \times N_{0}$ matrix given by

$$
\boldsymbol{b}=\left(\alpha \phi_{{ }_{u u}^{c}}^{c}-\beta \dot{\phi}_{u u}\right)\left(\stackrel{(1)}{u} \stackrel{(2)}{u} \ldots \stackrel{\left(N_{0}\right)}{u}\right) .
$$

Denote the $\left(N+N_{0}\right) \times\left(N+N_{0}\right)$ matrix of coefficients in (57) by $\boldsymbol{B}$. While this matrix is sparse, symmetric and nonsingular, it is not positive definite. It is therefore advisable in the solution of (57), to partition the matrix $\boldsymbol{B}$ into a positive definite submatrix that is factored using a standard skyline symmetric equation solver without pivoting, and then solve a condensed system of equations using a 
solver with pivoting. For this purpose $N_{0}$ control degrees of freedom are defined as for decomposition $A$, and the matrix $B$ is partitioned as

$$
\boldsymbol{B}=\left[\begin{array}{ll}
\boldsymbol{B}_{11} & \boldsymbol{B}_{12} \\
\boldsymbol{B}_{21} & \boldsymbol{B}_{22}
\end{array}\right], \quad \boldsymbol{B}_{11}=\phi_{{ }_{v \nu}}^{c}, \quad \boldsymbol{B}_{12}=\boldsymbol{B}_{21}^{\mathrm{t}}=\left[\begin{array}{ll}
\phi_{{ }^{\prime}}^{c} & \boldsymbol{b}_{\nu}
\end{array}\right], \quad \boldsymbol{B}_{22}=\left[\begin{array}{cc}
\phi_{{ }_{w w}}^{c} & \boldsymbol{b}_{w} \\
\boldsymbol{b}_{w}^{\mathrm{t}} & \mathbf{0}
\end{array}\right],
$$

where $\boldsymbol{b}_{v}$ and $\boldsymbol{b}_{w}$ are the rows of the matrix $\boldsymbol{b}$ corresponding to the passive and control degrees of freedom, respectively.

In solving (57), the passive components of the second order displacements (i.e. $\stackrel{(i i)}{\nu}$ ) are first eliminated to obtain a reduced system of $2 N_{0}$ equations in which the matrix of coefficients is

$$
\boldsymbol{C}=\boldsymbol{B}_{22}-\boldsymbol{B}_{21} \boldsymbol{B}_{11}^{-1} \boldsymbol{B}_{12}
$$

This reduced system is then solved using a routine with pivoting. The $\left(N-N_{0}\right) \times\left(N-N_{0}\right)$ matrix $\boldsymbol{B}_{11}$ can be factored using any standard solver without pivoting that exploits the symmetry and bandedness of the matrix. Evaluating $B_{11}^{-1} B_{12}$ then requires $N_{0}$ forward reductions and back substitutions. The factorization of $\boldsymbol{B}_{11}$ and $\boldsymbol{C}$, and forward reductions and back substitutions to calculate $\boldsymbol{B}_{11}^{-1} \boldsymbol{B}_{12}$ need only be performed once. They apply for the calculation of all $N_{0}\left(N_{0}+1\right) / 2$ second order displacement vectors ${ }^{(i j)} u$.

\subsection{Effect of the choice of $\hat{A}$ on results}

Two alternative decompositions, A and B have been outlined above. They differ in the choice of the complementary space $\hat{A}$. In both cases, the solutions can be derived in series form. However, different choices of the space $\hat{A}$ imply different parametrizations of the solutions. Therefore term by term equality between a series solution from decomposition $\mathbf{A}$ and its counterpart from decomposition $\mathrm{B}$ in general does not apply. All that can be said (assuming the series converge) is that if all terms are included, the solution sets are identical (although their parametrization would still differ). On the other hand, if (1) all modes are fully coincident, (2) the reference point coincides with the bifurcation point, (3) the same buckling modes $\tilde{u}$ are used for both decompositions, and (4) these modes span the nullspace of the tangent stiffness matrix $\phi^{c}{ }_{u u}$, then it is readily verified that the postbuckling coefficients $\phi_{i \varepsilon}, \phi_{i j \lambda}$ and $\phi_{i j k}$ will coincide. Furthermore, for a symmetric bifurcation (i.e. when all $\phi_{i j k}$ are zero), the fourth order coefficients $\phi_{i j k l}$ will also be identical in this case. Under other circumstances, the postbuckling coefficients from decompositions $\mathrm{A}$ and $\mathrm{B}$ will in general be different.

The assertions made above based on theoretical considerations have been confirmed by numerical examples involving a simple symmetric bifurcation. However, for the sake of brevity, a detailed account of these numerical examples is omitted. It was found that for decomposition A, the accuracy of the asymptotic results can deteriorate rapidly as the reference point was moved away from the bifurcation point, and is strongly dependent on the choice of the control degree(s) of freedom. Indeed, decomposition A can produce a nonzero postbuckling coefficient $\phi_{111}$ for a symmetric bifurcation, if the reference and bifurcation points do not coincide. Generally, these examples together with the possibility of obtaining higher order accuracy lead to the conclusion that decomposition B is preferable despite the somewhat larger amount of computational effort involved. Although decomposition B is also slightly more complicated, the programming effort need only be made once. It need not be repeated each time a new type of element is added to the program.

\section{Examples}

\subsection{Beam on nonlinear softening foundation (simple bifurcation)}

The problem of a beam on an elastic softening foundation with cubic nonlinearity is perhaps the simplest problem that can produce coincident or nearly coincident buckling modes, as well as symmetry breaking modal interaction. The potential energy for this problem can be written as 


$$
\phi(u, \lambda, \bar{u})=\int_{0}^{L}\left\{\frac{1}{2} u^{\prime \prime 2}+\frac{1}{2} u^{2}-\frac{1}{4} u^{4}-\frac{1}{2} \lambda\left(u^{\prime}+\bar{u}^{\prime}\right)^{2}-p u\right\} \mathrm{d} x,
$$

and the essential boundary condition used is $u^{\prime}=0$ at $x=0, L$. In $(61), u$ represents the transverse displacement, $\bar{u}$ is the initial imperfection in the form of a transverse displacement that exists when beam and foundation are stress free, $\lambda$ represents the axial compressive force in the beam, and $p$ is a uniform transverse load, which is held constant while the axial load $\lambda$ is increased. This transverse load was introduced so that the problem can exhibit symmetry breaking modal interaction (for $p=0$ the bifurcation is always symmetric and the second order displacements are zero). In all cases discussed below, the transverse load is taken as $p=5 / 6^{3 / 2}$, which produces a transverse displacement of $u(\lambda)=1 / \sqrt{6}$. Whereas the principal branch involves nonzero displacements, these displacements do not change with $\lambda$. Thus, $\dot{u}=0$.

Eigenmodes, orthonormalized to satisfy (47) with $\phi_{0}=L / 2$, and the corresponding bifurcation loads are given by

$$
\stackrel{i}{\tilde{u}}=\frac{L}{i \pi} \cos \left(\frac{i \pi x}{L}\right), \quad \lambda=\alpha^{2}\left[\left(\frac{\alpha L}{i \pi}\right)^{2}+\left(\frac{i \pi}{\alpha L}\right)^{2}\right]
$$

where $i$ is an integer representing the number of half-waves involved in the buckling mode, and $\alpha^{4}=1-3(u)^{2}=1 / 2$. For this example, the length is taken as $L=2^{1 / 4} \pi$ to minimize the buckling load for the $i=1$ mode. This produces a bifurcation load of $\lambda_{c}=\sqrt{2}$. Analytical evaluation of the postbuckling coefficients using the bifurcation point as the reference point about which the expansion is performed, and the complementary space $\hat{A}$ given in (52) (i.e. the one used for decomposition $B$ ) produces $\phi_{11}=\phi_{111}=0, \phi_{1111_{(i)}}=-47 \phi_{0}$, and $\phi_{1 \varepsilon}=-\lambda_{c} \phi_{0}$ for an imperfection shape that coincides with the buckling mode (i.e. $\overline{\bar{u}}=u$ ). Omitting all but the leading order terms, the reduced equilibrium equation, (33) or (46), becomes

$$
-\sqrt{2} \varepsilon-\xi \Delta \lambda-\frac{47}{6} \xi^{3}+\cdots=0
$$

where $\xi=\xi_{1}$ is the multiplier for the $i=1$ mode that arises in the decomposition of the displacements in the form of $(8)$.

For finite element discretization of the problem, 100 two-node elements of equal length based on cubic Hermite interpolation of the displacement $u$ are used. Integration of the element potential energy is done numerically, but with a sufficient number of Gaussian integration points to achieve exact integration. Postbuckling coefficients computed by the Iskfe program were found to be in excellent agreement (to within $10^{-6} \phi_{0}$ ) with the analytical values quoted above.

The bifurcated equilibrium branch for the perfect structure, and the relationship between load carrying capacity of the imperfect structure (load $\lambda$ at the first limit point) and the amplitude of the imperfection obtained from the leading order reduced equilibrium equation (63) are shown in Figs. 1 and 2 as dashed lines. For comparison, an 'exact' solution (continuous lines in Figs. 1 and 2) is computed numerically using the finite element discretization described above, and an incremental Newton technique, in which an approximation to the arclength along the equilibrium branch is controlled at every loadstep.

An improved asymptotic result for the imperfection sensitivity curve of Fig. 2 can be obtained by including $\phi_{1 \lambda \varepsilon} \Delta \lambda \varepsilon$ of (33) or (46) into (63). The resulting equilibrium condition,

$$
-(\sqrt{2}+\Delta \lambda) \varepsilon-\xi \Delta \lambda-\frac{47}{6} \xi^{3}+\cdots=0,
$$

together with the condition for a limit point $(d \lambda / d \xi=0)$ lead to a cubic equation, for which the solutions are shown in Fig. 2 as a dotted line. The improvement in accuracy is remarkable. At first sight, this may seem surprising, since the $\phi_{1 \lambda \varepsilon} \Delta \lambda \varepsilon$ term is only one of several higher order terms that would need to be included to guarantee higher order accuracy in the solution. However, on closer 


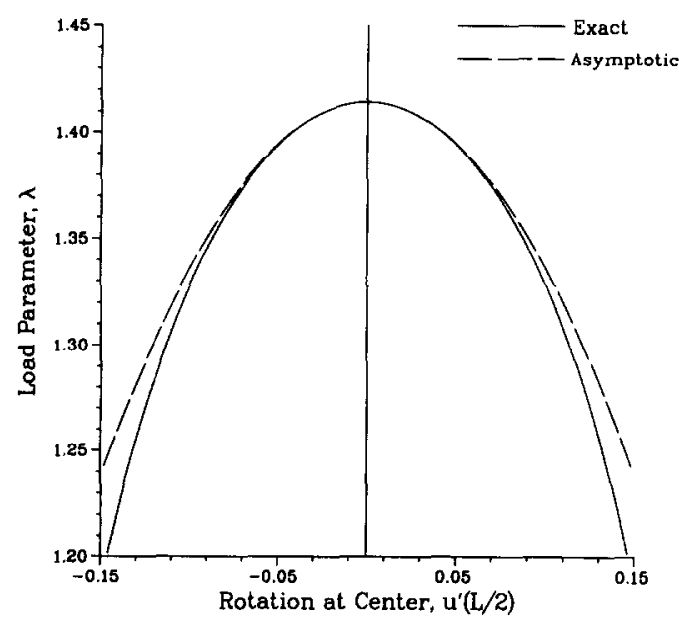

Fig. 1. Bifurcated equilibrium branch for a beam on an elastic foundation with simple bifurcation (Example 1).

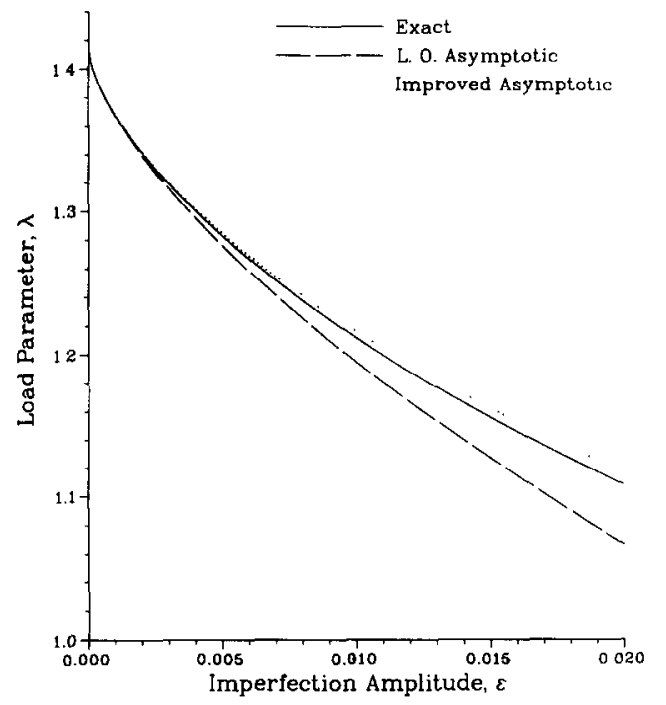

Fig. 2. Load-carrying capacity $\lambda$ as a function of the magnitude of imperfection $\varepsilon$ for a beam on an elastic foundation with simple bifurcation (Example 1).

examination, it is seen that a number of the other higher order terms are zero. Indeed it can be shown that

$$
\phi_{11111}=\phi_{111 \lambda}=\phi_{11 \varepsilon}=\phi_{111 \varepsilon}=0
$$

for this example. Another advantage of including this $\phi_{1 \lambda \varepsilon} \Delta \lambda \varepsilon$ term is that (at least for problems with linear prebuckling behavior) it precludes negative values of the load carrying capacity that would otherwise arise for a sufficiently large imperfection amplitude $\varepsilon$.

\subsection{Beam on elastic foundation (two coincident modes)}

If the length of the beam in the previous example is increased to $L=2^{3 / 4} \pi$ while all other problem parameters are kept the same, the $i=1$ and $i=2$ modes become coincident at $\lambda=5 / 2^{3 / 2}$.

The postbuckling coefficients were evaluated (i) analytically using the same approach as in the previous example, (ii) with the lskfe program using decomposition $B$, and (iii) with the lskfe program using decomposition $\mathrm{A}$.

The analytical values of the postbuckling coefficients are $\phi_{111}=\phi_{122}=\phi_{222}=0, \phi_{112}=(72)^{1 / 4} \phi_{0}$, $\phi_{1111}=-180 \phi_{0}, \phi_{1122}=-(19.2) \phi_{0}, \phi_{2222}=-(11.35) \phi_{0}, \phi_{1112}=\phi_{1222}=0$, and, for an imperfection of the shape $\overline{\bar{u}}$ given by (A.7) (Appendix A), $\phi_{i \varepsilon}=-\lambda_{c} \phi_{0} \overline{\bar{\xi}}_{i}$.

Bifurcation loads and postbuckling coefficients computed by the lskfe program using decomposition $B$, and 100 elements of equal size were again found to be in excellent agreement with the analytical values quoted above. (Errors are less than $10^{-7}$ for the bifurcation loads, and less than $10^{-6} \phi_{0}$ for the postbuckling coefficients. $)^{2}$

For decomposition $\mathrm{A}$, the displacement $u$ and rotation $u^{\prime}$ at the center node are used as the control degrees of freedom. Leading order postbuckling coefficients, $\phi_{i j k}$ and $\phi_{i \varepsilon}$ computed by the lskfe program are identical (within the accuracy of numerical computations, and the accuracy to which coincidence of the reference and bifurcation points was achieved) to those from decomposition $B$. However, as expected, the higher order coefficients $\phi_{i j k l}$ differ, with the results for decomposition $\mathrm{A}$ being $\phi_{1112}=\phi_{1222}=0$ (to within $10^{-8} \phi_{0}$ ), $\phi_{1111}=-362.0000 \phi_{0}, \phi_{1122}=-49.8667 \phi_{0}$, and $\phi_{2222}=$ $-11.3500 \phi_{0}$.

\footnotetext{
${ }^{2}$ Mode shapes computed numerically are in general a linear combination of the $i=1$ and $i=2$ modes. In order to meaningfully compare numerical and analytical postbuckling coefficients, it was necessary to redefine the basis for the nullspace of the tangent stiffness matrix such that mode 1 involves no displacement at the center node, and mode 2 involves no rotation at the center node.
} 
Other higher order postbuckling coefficients are not computed by the lskfe program, but, for this example, they can readily be evaluated analytically to obtain $\phi_{i \lambda \varepsilon}=-\phi_{0} \overline{\bar{\xi}}_{i}$, and $\phi_{i j \varepsilon}=\phi_{i j \lambda \lambda}=\phi_{i j k \lambda}=0$.

The following solutions for the bifurcated equilibrium branches for the perfect structure are shown in Figs. 3 and 5: (a) the solution computed as in the previous example by tracking the bifurcated equilibrium branches of the structure numerically (identified as 'Exact'), (b) the leading order asymptotic solution, obtained by solving (33), but considering only the leading order terms (identified as 'L.O. Asymptotic'), and (c), (d) improved asymptotic solutions (identified as 'Decomposition A' or ' $\mathrm{B}$ ' depending on which decomposition is used), obtained from a direct numerical solution of the reduced equilibrium equations that include the $\phi_{i j k l} \xi_{j} \xi_{k} \xi_{l}$ terms in addition to the leading order terms. (The reduced equilibrium equations were solved using essentially the same arclength control algorithm that was used for solution of the full system.)

Examine first the 'exact' solution shown in Fig. 3 from three orthogonal viewing directions. In addition to the principal equilibrium branch $\mathrm{AB}$, there are three bifurcated branches through the multiple bifurcation point $\mathrm{B}$. The asymmetric branch $\mathrm{G}^{\prime} \mathrm{BC}$, its mirror image, branch $\mathrm{GBC}$, and the symmetric branch LBI, which involves the $i=2$ mode only, and for which the rotation at the center $u^{\prime}(L / 2)$ is zero throughout. All bifurcated branches are seen to loop returning to the bifurcation point B. The equilibrium states are unstable on all bifurcated branches, with the number of negative eigenvalues being one on portion G'BCDEFE'D'C'BG of the asymmetric branch, and on portion BI of the symmetric branch, and two elsewhere on the bifurcated branches.

Secondary bifurcations occur at point $\mathrm{I}$, where the symmetric and asymmetric branches cross, and at points $\mathrm{F}$ and $\mathrm{K}$, which lie on an equilibrium branch $u=0.73129 \ldots$ for which there is no deformation of the beam for all values of the axial load $\lambda$. The deformed shapes of the beam corresponding to each of
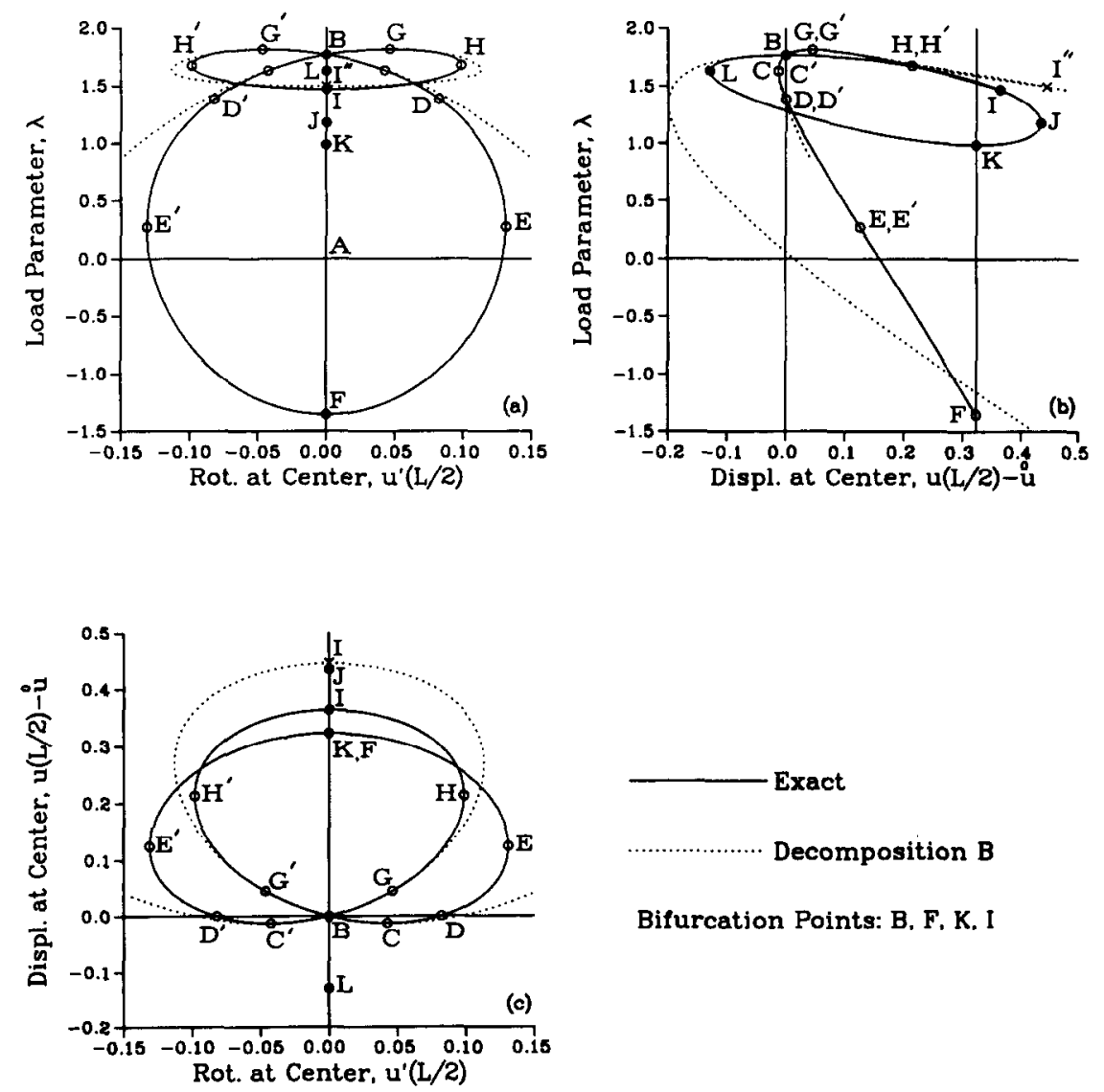

Bifurcation Points: B. F. K. I

Fig. 3. Bifurcation diagram for a beam on a nonlinear elastic foundation with multiple bifurcation (Example 2). 


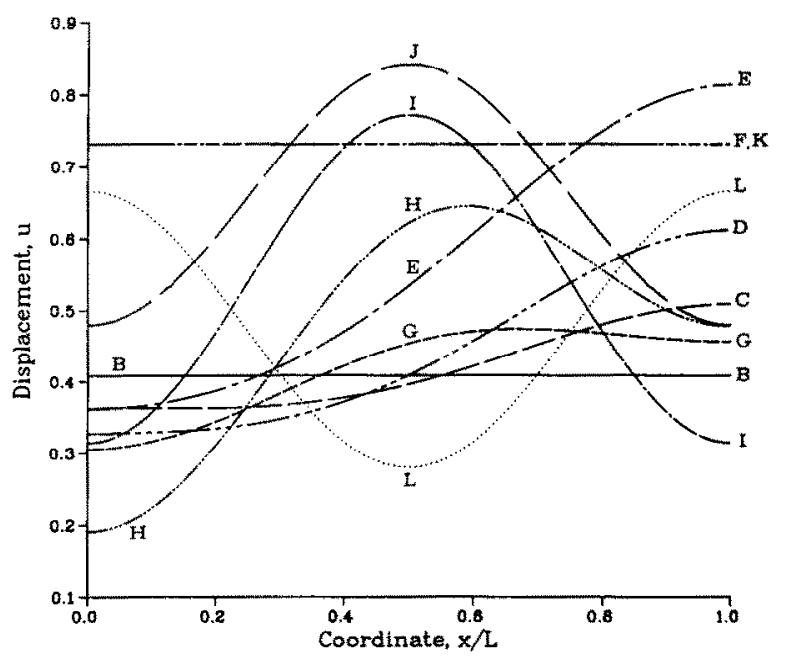

Fig. 4. Deformed shapes of a beam on a nonlinear elastic foundation with multiple bifurcation for states $A$ to $L$, as identified by labeled points in Fig. 3.
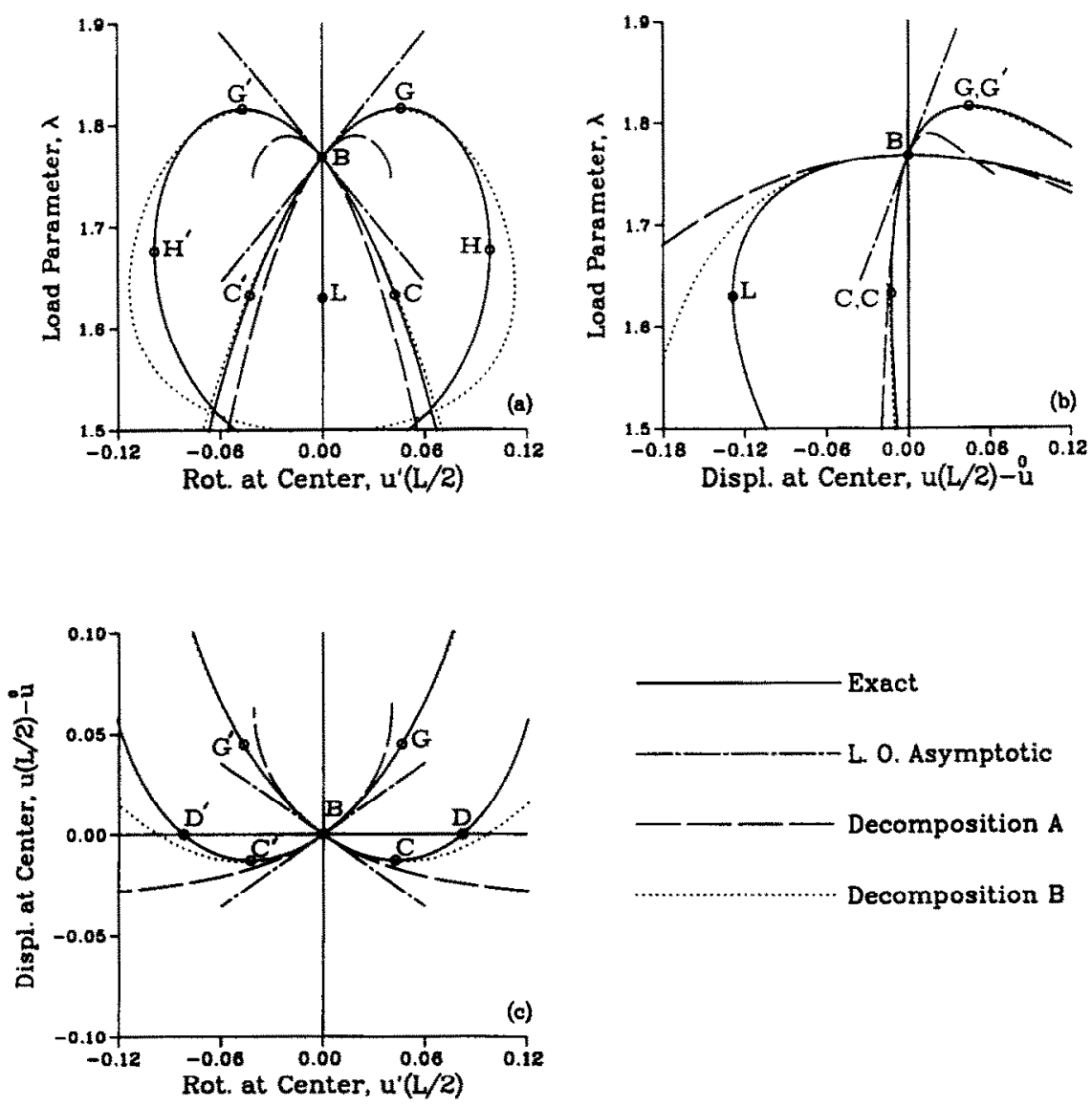

(c)

Fig. 5. Comparison of exact and asymptotic bifurcation diagrams for a beam on a nonlinear elastic foundation with multiple bifurcation (Example 2). 


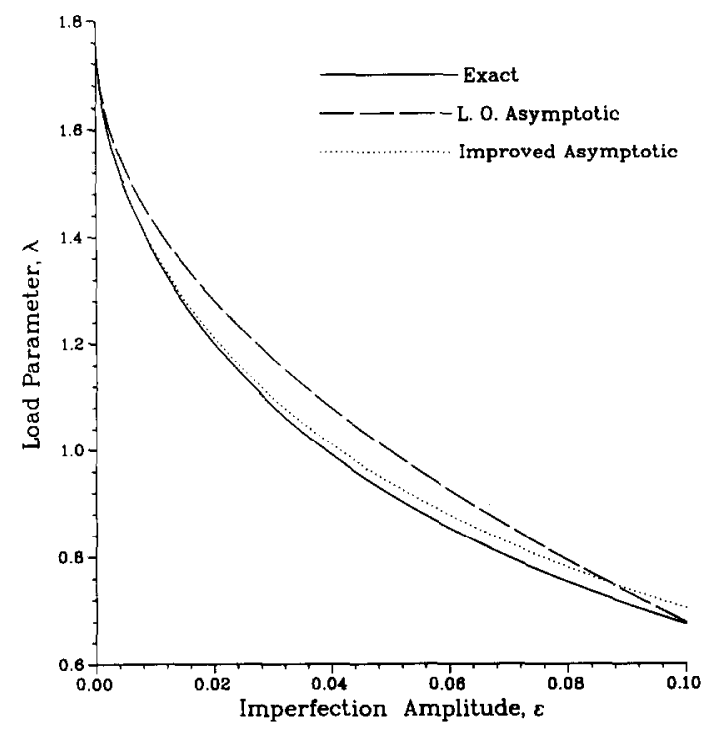

Fig. 6. Load-carrying capacity $\lambda$ as a function of the magnitude of imperfection $\varepsilon$ for a beam on an elastic foundation with multiple bifurcation and an imperfection of worst shape (Example 2).

the labeled points in Fig. 3 are shown in Fig. 4. Clearly the beam has a greater tendency to move towards increasing values of the displacement $u$, since this is the direction in which softening of the foundation occurs.

The comparison of the 'exact' solution, and the various asymptotic solutions is best examined in Fig. 5. This is a magnified version of the region close to the bifurcation point in Fig. 3. Therein, the leading order asymptotic solution is a straight line tangent to the exact solution at the bifurcation point, as expected.

Of the improved asymptotic solutions, that based on decomposition B (dotted line) is clearly far superior to that based on decomposition A (dashed line). Indeed, the improved asymptotic solution based on decomposition $A$ is no better than the leading order solution. The reason for this is that for decomposition $\mathrm{A}$, the postbuckling coefficients $\phi_{i j k \lambda}$ and $\phi_{i j \lambda \lambda}$ are in general nonzero, yet they are not included in the calculation of the improved asymptotic solution. As a result, the improved asymptotic solution for decomposition A does not possess higher order accuracy. On the other hand for decomposition B, the postbuckling coefficients $\phi_{i j k \lambda}$ and $\phi_{i j \lambda \lambda}$ are zero for this problem, so the improved asymptotic solution does possess higher order accuracy. As can be seen from Fig. 3, this higher order accurate asymptotic solution even reproduces the looping of the rising portion of the asymmetric bifurcated branch, as well as the secondary bifurcation at point $I^{\prime \prime}$, which corresponds to the secondary bifurcation at point I for the exact solution.

The load drop versus imperfection amplitude curve for an imperfection $\bar{u}=\varepsilon(\sqrt{2} \stackrel{(1)}{u}+\stackrel{(2)}{u}) / \sqrt{3}$ is shown in Fig. 6. This represents the worst shape of imperfection in the sense described in [10,11]. Again the leading order asymptotic solution is obtained from the truncated reduced equilibrium equation (only the first four terms in (33) or (46) are included). For the improved asymptotic solution, decomposition $B$ is used, and higher order accuracy is achieved by including all nonzero terms shown in (33) or (46).

\subsection{Shallow parabolic arch}

This example illustrates a problem involving a simple symmetric bifurcation, but with nonlinear prebuckling behavior. After nondimensionalization and application of shallow arch approximations, the potential energy for the shallow arch shown in Fig. 7 can be written as

$$
\phi(u, w, \lambda)=\int_{-1}^{1}\left\{\frac{1}{2}\left(u^{\prime}-\alpha x w^{\prime}+\frac{1}{2} w^{\prime 2}\right)+\frac{1}{2} w^{\prime \prime 2}+\lambda w\right\} \mathrm{d} x,
$$




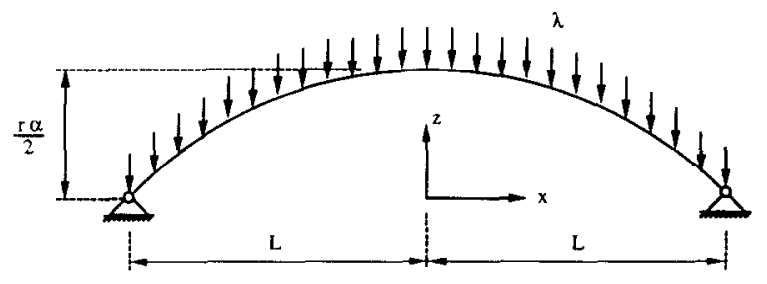

Fig. 7. Shallow arch (Example 3).

where $\alpha$ is a dimensionless depth parameter (the actual depth is $\alpha r / 2$, as shown in Fig. 7, where $r$ is the radius of gyration of the cross-section), $u$ and $w$ represent the displacement components in the $x$ and $z$ directions, respectively, and $\lambda$ represents the transverse load per unit length acting in the negative $z$ direction. All of these are nondimensionalized quantities. The actual values of the $x$ and $z$ components of displacement and the uniformly distributed transverse load are $u r^{2} / L, w r$ and $\lambda E A r^{3} / L^{4}$, respectively, where $E A$ is the axial rigidity and $L$ is the half span of the arch, as shown in Fig. 7 .

The solutions for the principal equilibrium branch and the bifurcated branches can readily be obtained analytically. The behavior depends on the depth parameter $\alpha$. For deeper arches $(\alpha>$ $9.185149 \ldots)$, bifurcation occurs before snapthrough. For shallower arches $(9.185149 \ldots>\alpha>$ 7.8290 ...) a limit point is encountered, followed by bifurcation on the falling portion of the principal branch; finally for $\alpha<7.8290 \ldots$ only snapthrough occurs with no possibility of a bifurcated solution. Here attention is focused on deeper arches for which bifurcation occurs before the limit point.

For the finite element solution of the problem a shear deformable element was used by replacing the potential energy function of (65) by

$$
\phi(u, w, \theta, \lambda)=\int_{-1}^{1}\left\{\frac{1}{2}\left(u^{\prime}+\alpha x w^{\prime}+\frac{1}{2} w^{\prime 2}\right)^{2}+\frac{1}{2} g\left(\theta+w^{\prime}\right)^{2}+\frac{1}{2} \theta^{\prime 2}+\lambda w\right\} \mathrm{d} x,
$$

where $\theta$ is the rotation of the cross-section, and $g$ is a penalty parameter (shear stiffness) taken as $g=10^{5}$ to allow the results from the finite element solution to be compared to the analytical solution based on the potential energy function of (65). Using 100 two-node elements with a single integration point and linear interpolation of $u, w$ and $\theta$, excellent agreement between the finite element and analytical solutions is achieved. (The two solutions could not be distinguished on a plot of the equilibrium branches.) A comparison of the leading order asymptotic solutions for the bifurcated equilibrium branches with these exact solutions is given in Fig. 8 for a variety of values of the depth

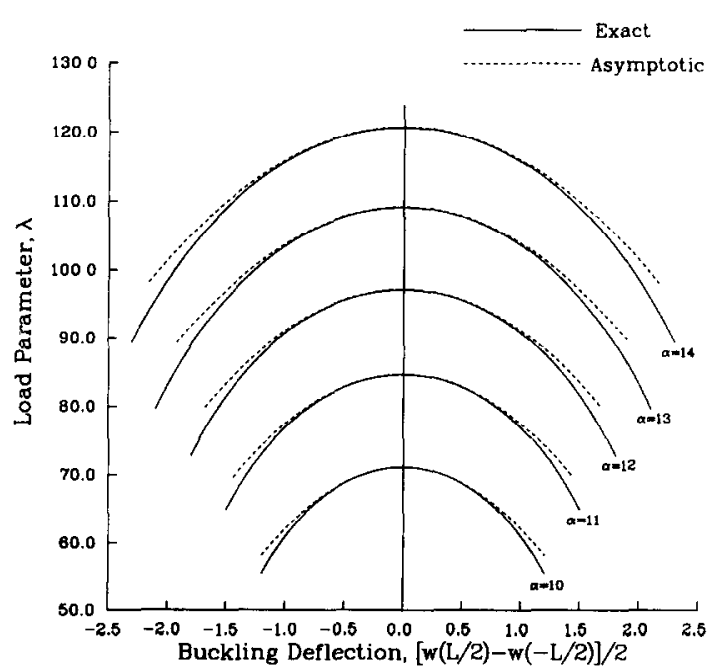

Fig. 8. Bifurcation diagrams for a shallow arch with various values of the dimensionless depth parameter $\alpha$.

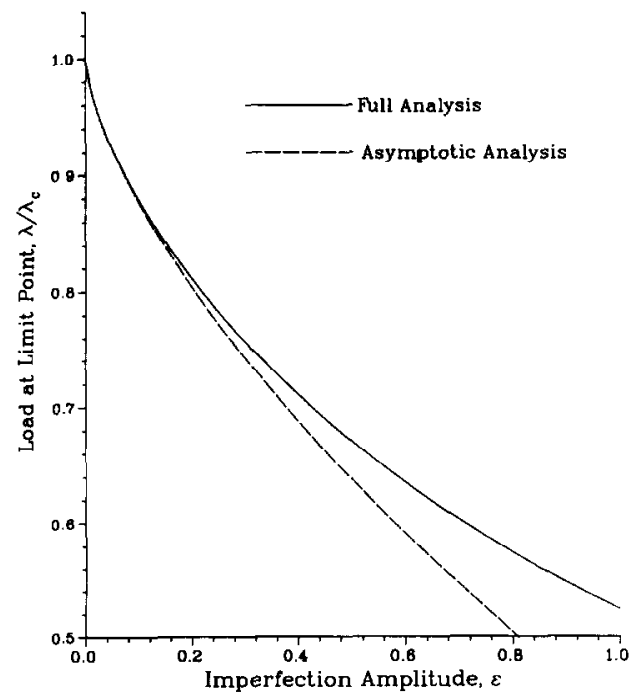

Fig. 9. Load carrying capacity of a shallow arch of depth $\alpha=12$ with a sinusoidal imperfection $\bar{w}=\varepsilon \sin (\pi x / L)$. 
parameter $\alpha$. As $\alpha \rightarrow 9.18149 \ldots$ a condition is approached in which the limit and bifurcation points become coincident. It is seen from Fig. 8 that this hardly affects the postbuckling bchavior or the range of validity of the asymptotic results. (The only problem encountered as $\alpha \rightarrow 9.18149 \ldots$ was calculating the displacement rate $\dot{u}$. Since $\dot{u}$ is rapidly varying, and becomes infinite as the limit point is approached, the above-described method of estimating $\dot{u}$ by extrapolation using the results from previous loadsteps only works if the size of the loadstep is small compared to the load increment to the limit point. On the other hand, direct calculation of $\dot{u}$ from (48) was found to be effective, even rather close to the bifurcation point.)

The drop in load carrying capacity for a sinusoidal imperfection $\bar{w}=\varepsilon \sin (\pi x / L)$ is shown in Fig. 8, for a value of the depth parameter of $\alpha=12$. Again good agreement between the asymptotic and exact results is obtained.

\section{Conclusions}

The Lyapunov-Schmidt-Koiter approach to investigate postbuckling behavior and imperfection sensitivity of structures has been generalized to include nonlinear prebuckling behavior as well as multiple, not necessarily coincident buckling modes. The asymptotic expansion of the reduced equilibrium equations is performed about a reference point on the principal equilibrium branch, which need not coincide with any of the bifurcation points. While this expansion is valid no matter whether the modes are coincident, closely spaced, or well separated, asymptotic solutions to these reduced equilibrium equations in the form of a truncated series are valid only sufficiently close to the reference point.

The method is implemented and evaluated for two different decompositions of the space of admissible displacements $A$. The first decomposition (decomposition A) bears some similarity to the 'equivalence transformation' approach of [26]. It represents essentially a nonlinear static condensation of all degrees of freedom except a few control degrees of freedom. The second decomposition (decomposition B) is a little more complicated to implement and requires some additional computation, but has the advantage that no corrections to the mode shapes are required, and a number of the higher order postbuckling coefficients vanish for most (if not all) problems with linear prebuckling behavior.

A number of examples, including comparisons of the asymptotic and exact results illustrate the effectiveness and limitations of the method.

\section{Acknowledgment}

This work was supported by the National Science Foundation as Grant No. MSS-9103227 and CES-8857002.

\section{Appendix A}

For a wide variety of problems with geometric nonlinearities only and linear prebuckling behavior, the potential energy can be written as

$$
\phi(u, \lambda, \bar{u})=W(e)-\lambda P u,
$$

where $W(\cdot)$ is the strain energy function, which is assumed to be quadratic in the strains $e$ (i.e. $\left.W_{\text {eee }}=0\right) ; P$ is a linear operator representing the loading pattern, and the strains $e$ can be written as

$$
e=b u+c(\bar{u}, u)+\frac{1}{2} c(u, u),
$$

where $b$ is a linear operator, $c(\cdot, \cdot)$ is a bilinear and symmetric operator, and $\bar{u}$ represents the initial 
displacements at which the structure is stress free. Equations (A.1) and (A.2) apply, for example for the Donnel-Von Kárman-Vlasov shallow shell theory, provided that the prebuckling solution involves constant transverse displacement. Furthermore, it is assumed that the prebuckling solution for the perfect structure can be written as $u=\lambda \dot{u}$ where $\dot{u}$ is constant, and

$$
c(\dot{u}, \delta u)=0 \quad \forall \delta u \in A
$$

For such a structure with linear prebuckling behavior, the expressions for the postbuckling coefficients reduce to

where

$$
\begin{array}{ll}
\phi_{i j}=W_{e e} e^{(i)} \stackrel{(j)}{e}+\lambda_{c} \phi_{i j \lambda}, & \phi_{i j \lambda}=W_{e e} \dot{e} c(\stackrel{(i)}{u}, \stackrel{(j)}{u}), \\
\phi_{i j k}=a_{i j k}+a_{j k i}+a_{k i j}, & \phi_{i \varepsilon}=\lambda_{c} \dot{\phi}_{u u} \overline{\bar{u}} \stackrel{(i)}{u}=\lambda_{c} W_{e e} \dot{e} c(\overline{\bar{u}}, \stackrel{(i)}{u}),
\end{array}
$$

$$
\dot{e} \equiv b \dot{u}, \quad \stackrel{(i)}{e} \equiv b \stackrel{(i)}{u}, \quad a_{i j k}=W_{e e} \stackrel{(i)}{e c}(\stackrel{(j)}{u}, \stackrel{(k)}{u})
$$

Furthermore, if decomposition B is used, then $\phi_{i j k \lambda}=\phi_{i j \lambda \lambda}=0$. Finally, if, in addition to linear prebuckling, and the use of decomposition $B$, the imperfection shape $\overline{\bar{u}}$ is a linear combination of the modes in the form

$$
\overline{\bar{u}}=\sum_{i} \overline{\bar{\xi}}_{i} \stackrel{(i)}{u},
$$

then

$$
{ }^{(\varepsilon)} u=0, \quad \phi_{i \varepsilon}=\lambda_{c} \phi_{i \varepsilon \lambda}=\sum_{j} \phi_{i j \lambda} \overline{\bar{\xi}}_{j}, \quad \phi_{i j \varepsilon}=\sum_{k}\left(a_{i j k}+a_{j i k}\right) \overline{\bar{\xi}}_{k}
$$

\section{References}

[1] W.T. Koiter, The stability of elastic equilibrium, Thesis, Technische Hooge School at Delft (in Dutch), English Translation, 1945; Technical Report No. AFFDL-TR-70-25, sponsored by Lockheed Missiles and Space Co., 1970.

[2] W.T. Koiter, The effect of axisymmetric imperfections on the buckling of cylindrical shells under axial compression, Koninkl, Ned. Akad. Wetenschap. Proc., B66 (1963) 265-279.

[3] V.I. Weingarten, E.J. Morgan and P. Seide, Final report on development of design criteria for elastic stability of thin shell structures, STL/TR-60-0000-19426, Space Technology Laboratories, Inc., Los Angeles, CA, 1960.

[4] V.I. Weingarten, E.J. Morgan and P. Seide, Elastic stability of thin-walled cylindrical and conical shells under combined internal pressure and axial compression, AIAA J. 3 (1965) 1118-1125.

[5] N. Yamaki, Elastic Stability of Circular Cylindrical Shells (North-Holland, Amsterdam, 1984).

[6] H.B. Keller, Numerical solution of bifurcation and nonlinear eigenvalue problems, in: P.H. Rabinowitz, ed., Applications of Bifurcation Theory (Academic Press, New York, 1977) 359-384.

[7] E. Riks, The application of Newton's method to the problem of elastic stability, J. Appl. Mech. 39 (1972) $1060-1066$.

[8] E. Riks and C.C. Rankin, Bordered equations in continuation methods: An improved solution technique, NLR MP 87057U, National Aerospace Laboratory, The Netherlands, 1987.

[9] D. Ho, Buckling load of nonlinear systems with multiple eigenvalues, Internat. J. Solids and Structures 10 (1974) $1315-1330$.

[10] W.T. Koiter, Current trends in the theory of buckling, in: B. Budiansky, ed., Symposium on Buckling of Structures, sponsored by the International Union of Theoretical and Applied Mechanics (IUTAM), Harvard University, Cambridge, MA, 1974 (Springer, Berlin, 1976).

[11] N. Triantafyllidis and R. Peek, On stability and the worst imperfection shape in solids with nearly simultaneous eigenmodes, Internat. J. Solids and Structures 29 (18) (1992) 2281-2299.

[12] J.C. Amazigo, B. Budiansky and G.F. Carrier, Asymptotic analysis of the buckling of imperfect columns on nonlinear elastic foundations, Internat. J. Solids and Structures 6 (1970) 1341-1356.

[13] B. Budiansky, Theory of buckling and postbuckling behavior of elastic structures, Adv. Appl. Mech. 14 (1974) 1-65.

[14] D. Hui and I.H.Y. Du, Initial postbuckling behavior of imperfect, antisymmetric crossply cylindrical shells under torsion, J. Appl. Mech. 54 (1987) 174-180.

[15] J.W. Hutchinson and J.C. Amazigo, Imperfection sensitivity of eccentrically stiffened cylindrical shells, AIAA J. 5 (1967) $392-401$. 
[16] J.W. Hutchinson and J.C. Frauenthal, Elastic postbuckling behavior of stiffened and barreled cylindrical shells, J. Appl. Mech. 36 (1969) 784-790.

[17] N. Rizzi and A. Tatone, Symbolic manipulation in buckling and postbuckling analysis, Comput. \& Structures 21 (1985) 691-700.

[18] N.P. Semenyuk, Stability of imperfectly cylindrical shells made from composite materials, Prikl. Mekh. 23 (1987) 37-43; Translation in: Soviet Applied Mechanics (Plenum, New York).

[19] E. Carnoy, Postbuckling analysis of elastic structures by the finite element method, Comput. Methods Appl. Mech. Engrg. 23 (1980) 143-174.

[20] R.L. Citerley, Computer programs for imperfection sensitivity analysis of stiffened cylindrical shells, Weld. Res. Counc. Bull. 313 (1986).

[21] G.A. Cohen, User document for computer programs for ring-stiffened shells of revolution, NASA CR-2086, 1973.

[22] G.A. Cohen and R.T. Haftka, Sensitivity of buckling loads of anisotropic shells of revolution to geometric imperfections and design changes, Comput. \& Structures 31 (1989) 985-995.

[23] R.T. Haftka, A Koiter-type method for finite element analysis of nonlinear structural behavior, Ph.D. Thesis, The University of California, San Diego, La Jolla, CA, 1970.

[24] T.J. Healy, A group theoretic approach to computational bifurcation problems with symmetry, Comput. Methods Appl. Mech. Engrg. 67 (1988) 257-295.

[25] C.C. Rankin and F.A. Brogan, Application of the Thurston bifurcation solution strategy to problems with modal interaction, Proc. AIAA (April 1988) No. 88-2286.

[26] G.A. Thurston, F.A. Brogan and P. Stehlin, Postbuckling analysis using a general purpose code, AIAA J. 24 (1986) 1013-1020.

[27] E. Byskov and J.W. Hutchinson, Mode interaction in axially stiffened cylindrical shells, AIAA J. 15 (1977) 941-948.

[28] J.M.T. Thompson and G.W. Hunt, A General Theory of Elastic Stability (Wiley, London, 1973).

[29] G.H. Golub, Matrix Computations (Johns Hopkins Univ. Press, Baltimore, MD, 1989). 